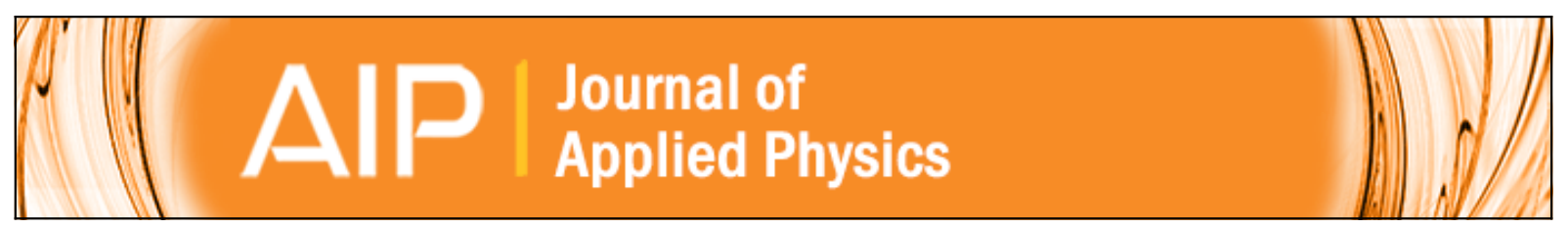

\title{
Helicity sensitive terahertz radiation detection by dual-grating-gate high electron mobility transistors
}

P. Faltermeier, P. Olbrich, W. Probst, L. Schell, T. Watanabe, S. A. Boubanga-Tombet, T. Otsuji, and S. D. Ganichev

Citation: Journal of Applied Physics 118, 084301 (2015); doi: 10.1063/1.4928969

View online: http://dx.doi.org/10.1063/1.4928969

View Table of Contents: http://scitation.aip.org/content/aip/journal/jap/118/8?ver=pdfcov

Published by the AIP Publishing

\section{Articles you may be interested in}

Plasmon dispersions in high electron mobility terahertz detectors

Appl. Phys. Lett. 104, 263514 (2014); 10.1063/1.4886970

Ultrahigh sensitive sub-terahertz detection by InP-based asymmetric dual-grating-gate high-electron-mobility transistors and their broadband characteristics

Appl. Phys. Lett. 104, 251114 (2014); 10.1063/1.4885499

Nonlinear photoresponse of field effect transistors terahertz detectors at high irradiation intensities J. Appl. Phys. 115, 164514 (2014); 10.1063/1.4872031

Helicity sensitive terahertz radiation detection by field effect transistors

J. Appl. Phys. 111, 124504 (2012); 10.1063/1.4729043

Terahertz radiation detection by field effect transistor in magnetic field

Appl. Phys. Lett. 95, 072106 (2009); 10.1063/1.3207886

The new SR865 2 MHz Lock-In Amplifier ... \$7950

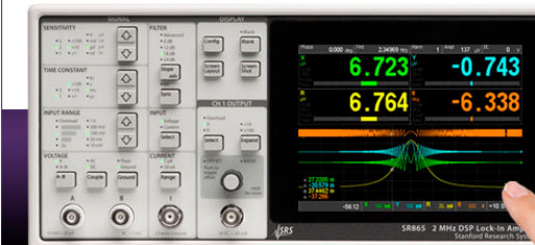

SRS Stanford Research Systems

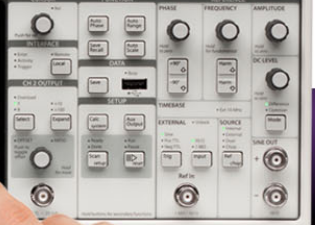

()

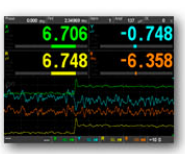

Chart recording

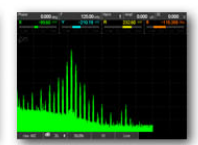

FFT displays

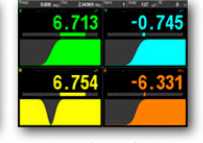

Trend analysis
Intuitive front-panel operation Touchscreen data display Embedded web server and iOS app - Synch multiple SR865s via $10 \mathrm{MHz}$ timebase I/O . View results on a TV or monitor (HDMl output)
. $1 \mathrm{mHz}$ to $2 \mathrm{MHz}$

$.2 .5 \mathrm{nV} / \sqrt{ } \mathrm{Hz}$ input noise . $1 \mu$ s to $30 \mathrm{ks}$ time constants $\cdot 1.25 \mathrm{MHz}$ data streaming rate - Sine out with DC offset - GPIB, RS-232, Ethernet \& USB 


\title{
Helicity sensitive terahertz radiation detection by dual-grating-gate high electron mobility transistors
}

\author{
P. Faltermeier, ${ }^{1}$ P. Olbrich, ${ }^{1}$ W. Probst, ${ }^{1}$ L. Schell, ${ }^{1}$ T. Watanabe, ${ }^{2}$ S. A. Boubanga-Tombet, ${ }^{2}$ \\ T. Otsuji, ${ }^{2}$ and S. D. Ganichev ${ }^{1}$ \\ ${ }^{1}$ Terahertz Center, University of Regensburg, 93040 Regensburg, Germany \\ ${ }^{2}$ Research Institute of Electrical Communication, Tohoku University, 980-8577 Sendai, Japan
}

(Received 7 July 2015; accepted 10 August 2015; published online 24 August 2015)

\begin{abstract}
We report on the observation of a radiation helicity sensitive photocurrent excited by terahertz (THz) radiation in dual-grating-gate (DGG) InAlAs/InGaAs/InAlAs/InP high electron mobility transistors (HEMT). For a circular polarization, the current measured between source and drain contacts changes its sign with the inversion of the radiation helicity. For elliptically polarized radiation, the total current is described by superposition of the Stokes parameters with different weights. Moreover, by variation of gate voltages applied to individual gratings, the photocurrent can be defined either by the Stokes parameter defining the radiation helicity or those for linear polarization. We show that artificial non-centrosymmetric microperiodic structures with a twodimensional electron system excited by $\mathrm{THz}$ radiation exhibit a $d c$ photocurrent caused by the combined action of a spatially periodic in-plane potential and spatially modulated light. The results provide a proof of principle for the application of DGG HEMT for all-electric detection of the radiation's polarization state. (C) 2015 AIP Publishing LLC. [http://dx.doi.org/10.1063/1.4928969]
\end{abstract}

\section{INTRODUCTION}

Field-effect-transistors (FETs) have emerged as promising devices for sensitive and fast room temperature detection of terahertz $(\mathrm{THz})$ radiation. ${ }^{1,2}$ They are considered as a good candidate for real-time $\mathrm{THz}$ imaging and spectroscopic analysis, ${ }^{3,4}$ as well as future $\mathrm{THz}$ wireless communications. ${ }^{5}$ Devices employing plasmonic effects in FETs have already been applied for room temperature detection of radiation with frequencies from tens of $\mathrm{GHz}$ up to several $\mathrm{THz}$ and enable the combination of individual detectors in a matrix. They are characterized by high responsivity (up to a few $\mathrm{kV} / \mathrm{W}$ ), low noise equivalent power (down to $10 \mathrm{pW} / \sqrt{\mathrm{Hz}}$ ), fast response time (tens of picoseconds), and large dynamic range (linear power response up to $10 \mathrm{~kW} / \mathrm{cm}^{2}$ ), see, e.g., Refs. 2 and 6-11. The operation principle of FET THz detectors used so far is based on the nonlinear properties of the two-dimensional (2D) plasma in the transistor channel. The standard Dyakonov-Shur model ${ }^{12}$ assumes that radiation is coupled to the transistor by an effective antenna, which generates an $a c$ voltage predominantly on one side of the transistor. Both resonant ${ }^{13}$ and non-resonant ${ }^{14}$ regimes of $\mathrm{THz}$ detection have been studied. While research aimed to development of THz FET detectors is focused on single gate structures, recently several groups have shown that higher sensitivities are expected for structures with periodic symmetric and asymmetric metal stripes or gates. ${ }^{9,15-22}$ In particular, dual-grating-gate (DGG) FET are considered as a good candidate for sensitive $\mathrm{THz}$ detection. The first data obtained on dual-gated-structures demonstrated a substantial enhancement of the photoelectric response and an ability to control detector parameters by variation of individual gate bias voltage. ${ }^{9}$ At the same time, THz electric field applied to FETs with asymmetric periodic dual gate structure is expected to give rise to electronic ratchet effects ${ }^{20,23-26}$ (for review, see Ref. 24) and plasmonic ratchet effects. ${ }^{27}$ Besides improving the figure of merits of FET detectors, ratchet effects may also result in new functionalities. In particular, they may induce photocurrents driven solely by the radiation helicity.

Here, we report on the observation of a radiation helicity sensitive photocurrent excited by $\mathrm{THz}$ radiation in dualgrating-gate InAlAs/InGaAs/InAlAs/InP high electron mobility transistors (HEMT). We show that artificial non-centrosymmetric microperiodic structures with a twodimensional electron system excited by $\mathrm{THz}$ radiation exhibit a $d c$ photocurrent caused by the lateral asymmetry of the applied static potential and terahertz electric field. We demonstrate that depending on gate voltages applied to the individual gratings of the dual-grating-gate, the response can be proportional to either the Stokes parameters ${ }^{28}$ defining the radiation helicity or those for linear polarization. As an important result, for a wide range of gate voltages, we observed a photocurrent $j_{\mathrm{C}}$ being proportional to the radiation helicity $P_{\text {circ }}=\left(I_{\sigma^{+}}-I_{\sigma^{-}}\right) /\left(I_{\sigma^{+}}+I_{\sigma^{-}}\right)$, where $I_{\sigma^{+}}$and $I_{\sigma^{-}}$are intensities of right- and left-handed circularly polarized light. For the circular photocurrent, $j_{\mathrm{C}}$ measured between source and drain contacts changes its sign with the inversion of the radiation helicity. This observation is of particular importance for a basic understanding of plasmon-photogalvanic and quantum ratchet effects. It also has a large potential for the development of an all-electric detector of the radiation's polarization state, which was so far realized applying less sensitive photogalvanic effects only. ${ }^{29-32}$ The observed phenomena is discussed in the framework of electronic ratchet ${ }^{20,22,24-26}$ and plasmonic ratchet effects excited in a 2D electron system with a spatially periodic $d c$ in-plane potential. ${ }^{9,22,27}$ 


\section{EXPERIMENTAL TECHNIQUE}

The device structure is based on an InAlAs/InGaAs/ InAlAs/InP (HEMT) and incorporates doubly inter-digitated grating gates (DGG) $G_{1}$ and $G_{2}$. A sketch and a photograph of the gates are shown in Fig. 1(a) and inset in Fig. 1(b). The $2 \mathrm{D}$ electron channel is formed in a quantum well (QW) at the heterointerface between a $16 \mathrm{~nm}$-thick undoped InGaAs composite channel layer and a $23 \mathrm{~nm}$-thick, Si-doped InAlAs carrier-supplying layer. The electron density of the 2DEG is about $3 \times 10^{12} \mathrm{~cm}^{2}$, electron effective mass normalized on free-electron mass $m_{0}$ and room temperature Hall mobility are $m / m_{0}=0.04$ and $\mu_{0}=11500 \pm 500 \mathrm{~cm}^{2} /(\mathrm{Vs})$, respectively. The latter value has been obtained by noncontact Hall measurement. ${ }^{33}$ The DGG gate is formed with $65 \mathrm{~nm}$-thick Ti/Au/Ti by a standard lift-off process. The footprint of the narrower gate fingers $G_{1}$ was defined by an Ebeam lithography, whereas that of the wider gate fingers $G_{2}$ was defined by a photolithography. In all studied structures, the metal fingers of the grating gates $G_{1}$ and $G_{2}$ have the same length, being $d_{\mathrm{G} 1}=200 \mathrm{~nm}$ and $d_{\mathrm{G} 2}=800 \mathrm{~nm}$. The spacing between narrow and wide DGG fingers is asymmetric with $a_{\mathrm{G} 1}=200 \mathrm{~nm}$ and $a_{\mathrm{G} 2}=400 \mathrm{~nm}$ (see Fig. 1). The size of the active area, covered with the grating, is about $20 \mu \mathrm{m} \times 20 \mu \mathrm{m}$. The source/drain ohmic contacts were made in a non-alloyed way with $n+\operatorname{InGaAs}(15 \mathrm{~nm}) / n+\operatorname{InAlAs}$ $(15 \mathrm{~nm})$ cap layers and Ti $/ \mathrm{Pt} / \mathrm{Au}$ metals. ${ }^{34}$ The typical value

(a)

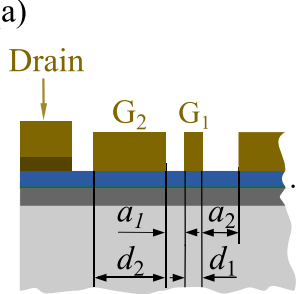

(b)

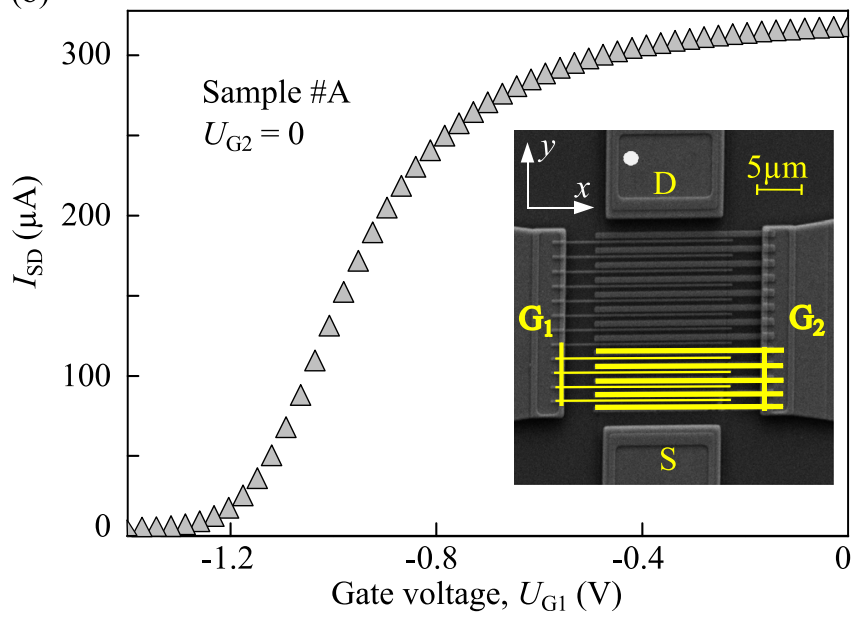

$15 \mathrm{~nm} \mathrm{In}_{0.53} \mathrm{GaAs}, \mathrm{n}++$

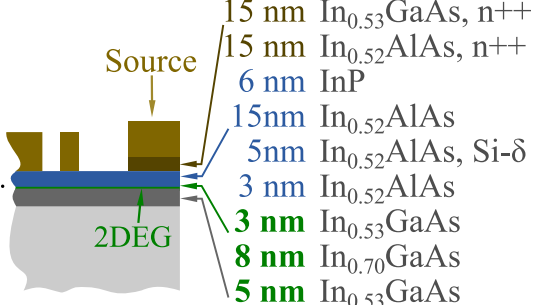

5 nm $\mathrm{In}_{0.53} \mathrm{GaAs}$ $100 \mathrm{~nm} \operatorname{In}_{0.52} \mathrm{AlAs}$

FIG. 1. (a) Sketch of the dual-grating-gate HEMT. Cross-section of the structure shows the layer sequence and indicates the width of the fingers $\left(d_{1 / 2}\right)$ and the fingers spacings $\left(a_{1 / 2}\right)$. THz radiation at $2.54 \mathrm{THz}$ is applied at normal incidence. (b) Drain-to-source current as a function of the gate voltage $U_{\mathrm{G} 1}$ measured at $U_{\mathrm{G} 2}=0 \mathrm{~V}$. Inset shows the photograph of the structure. Here, $\mathrm{G}_{1} / \mathrm{G}_{2}, \mathrm{~S}$, and $\mathrm{D}$ denote first/second gate, source, and drain, respectively. Part of $\mathrm{G}_{1} / \mathrm{G}_{2}$ structure is highlighted by yellow lines for visualization. of our samples is $0.07-0.09 \Omega-\mathrm{mm}$ (the length in the unit is the width of the contact pad). The axis along the gate's fingers is denoted as $x$ and that along source and drain as $y$. The characteristic source/drain current-gate voltage dependence obtained by transport measurement is shown for sample \#A in Fig. 1(b).

All experiments are performed at room temperature. The HEMT structures were illuminated with polarized $\mathrm{THz}$ and microwave (MW) radiation at normal incidence. For optical excitation, we used low power $c w$ optically pumped $\mathrm{CH}_{3} \mathrm{OH} \mathrm{THz}$ laser ${ }^{35,36}$ and Gunn diodes providing monochromatic radiation with frequencies $f=2.54 \mathrm{THz}$ and $95.5 \mathrm{GHz}$, respectively. The radiation peak power $P$, being of the order of several milliwatts at the sample's position, has been controlled by pyroelectric detectors and focused onto samples by parabolic mirrors ( $\mathrm{THz}$ laser) or horn antenna (Gunn diode). The spatial beam distribution of $\mathrm{THz}$ radiation had an almost Gaussian profile, checked with a pyroelectric camera. ${ }^{37-39} \mathrm{THz}$ laser radiation peak intensity, $I$, for laser spot being of about $1.2 \mathrm{~mm}$ diameter on the sample, was $I \approx 8 \mathrm{~W} / \mathrm{cm}^{2}$. The profile of the microwave radiation and, in particular, the efficiency of the radiation coupling to the sample could not be determined with satisfactory accuracy. Thus, all microwave data are given in arbitrary units. The polarization state of $\mathrm{THz}$ radiation has been varied applying crystal quartz $\lambda / 4$ - or $\lambda / 2$-plates. ${ }^{40}$ To obtain circular and elliptically polarized light the quarter-wave plate was rotated by the angle, $\varphi$, between the initial polarization plane and the optical axis of the plate. The radiation polarization states for several angles $\varphi$ are illustrated on top of Fig. 2. Orientation of the linearly polarized radiation is defined by the azimuth angle $\alpha$, with $\alpha=\varphi=0$ chosen in such a way that the electric field of incident linearly polarized light is directed along $x$-direction. Different orientations of linearly polarized MW radiation were obtained by rotation of a metal wire grid polarizer. The photocurrent excited between source and drain is measured across a $50 \Omega$ load resistor applying the standard lock-in technique.

\section{PHOTOCURRENT EXPERIMENT}

Illuminating the structure with elliptically (circular) polarized radiation of terahertz laser operating at frequency $f=2.54 \mathrm{THz}$, we observed a $d c$ current strongly depending on the radiation polarization. Figure 2(a) shows the photocurrent as a function of the phase angle $\varphi$ defining the radiation polarization state. The data are obtained for zero gate voltage at the gate $2, U_{\mathrm{G} 2}=0$ and $U_{\mathrm{G} 1}=-1.06 \mathrm{~V}$. The principal observation is that for right- $\left(\sigma^{+}\right)$and left-handed $\left(\sigma^{-}\right)$ polarizations, i.e., for $\varphi=45^{\circ}$ and $135^{\circ}$, the signs of the photocurrent $j_{y}$ are opposite. The overall dependence $j_{y}(\varphi)$ is well described by

$$
j_{y}(\varphi)=j_{0} s_{0}+j_{1} s_{1}(\varphi)+j_{2} s_{2}(\varphi)+j_{C} s_{3}(\varphi)
$$

and corresponds to the superposition of the Stokes parameters with different weights given by the coefficients $j_{0}, j_{1}, j_{2}$, and $j_{\mathrm{C}}$, which in the experimental geometry applying rotation of quarter-wave plate the Stokes parameters change after 


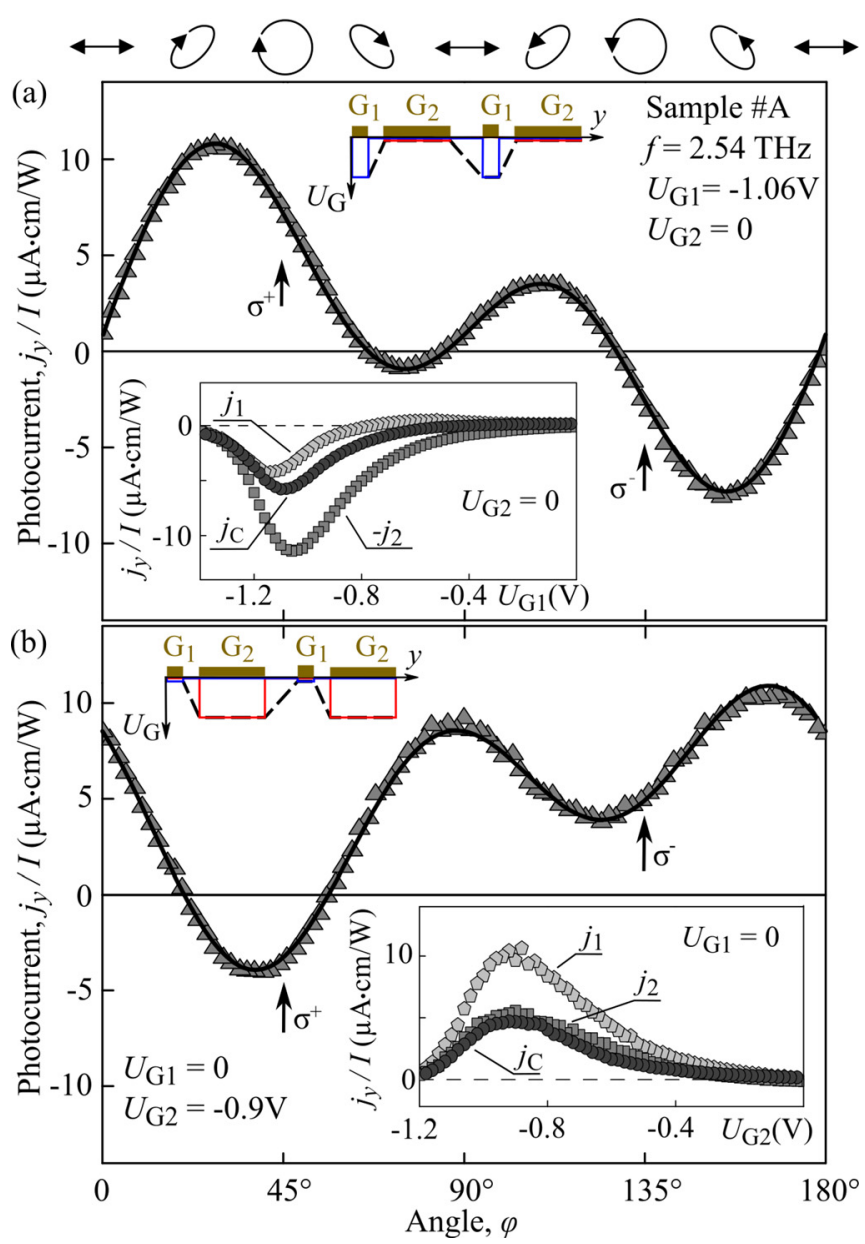

FIG. 2. THz radiation induced normalized photocurrent $j_{y} / I$ as a function of the angle $\varphi$ defining the radiation helicity. The current is measured for different voltages applied to the first and second gates. (a) The data for $U_{\mathrm{G} 1}=-1.06 \mathrm{~V}$ at gate 1 and zero gate voltage at gate 2 . (b) The photocurrent measured for zero gate voltage at gate 1 and $U_{\mathrm{G} 2}=-0.9 \mathrm{~V}$. Full lines show fits to the total current calculated after Eq. (1). The ellipses on top illustrate the polarization states for various $\varphi$. Insets show amplitudes of photocurrent contributions $j_{\mathrm{C}} / I$, driven by the light helicity, and $j_{1} / I\left(j_{2} / I\right)$, induced by linear polarization, as a function of the gate voltages $U_{\mathrm{G} 1}$ or $U_{\mathrm{G} 2}$. Second set of the insets schematically show corresponding gate potentials. Dashed lines are guide for the eye indicating the potential asymmetry in $y$-direction. Note that presence of the metal gates results in a nonzero potential even for $U_{\mathrm{G}}=0$.

$$
\begin{gathered}
s_{0} \equiv\left|E_{0 x}\right|^{2}+\left|E_{0 y}\right|^{2}, \\
s_{1} \equiv\left(\left|E_{0 x}\right|^{2}-\left|E_{0 y}\right|^{2}\right) / s_{0}=\frac{\cos 4 \varphi+1}{2}, \\
s_{2} \equiv\left(E_{0 x} E_{0 y}^{*}+E_{0 x}^{*} E_{0 y}\right) / s_{0}=\frac{\sin 4 \varphi}{2}, \\
s_{3} \equiv i\left(E_{0 x} E_{0 y}^{*}-E_{0 x}^{*} E_{0 y}\right) / s_{0}=-P_{\text {circ }}=-\sin 2 \varphi .
\end{gathered}
$$

Here, $\boldsymbol{E}_{0}$ is the radiation electric field in vacuum, $s_{0}$ determines the radiation intensity, $s_{1}$ and $s_{2}$ define the linear polarization of radiation in the $(x y)$ and rotated by $45^{\circ}$ coordinate frames, and $s_{3}$ describes the degree of circular polarization or helicity of radiation. The latter one vanishes for linearly polarized light. Consequently, individual photocurrent contributions in Eq. (1) are induced by unpolarized, linearly or circularly polarized light components. While the polarization dependence given by Eq. (1) has been detected for arbitrary relations between voltages applied to the first and second gates, the magnitude and even the sign of the individual contributions can be controlled by the gate voltages. The inset in Fig. 2(a) shows a gate dependence of the polarization dependent contributions to the total photocurrent. ${ }^{41}$ The dependence on the gate voltage $U_{\mathrm{G} 1}$ is obtained for zero biased second gate. Photocurrent measured in the close circuit configuration with $R_{L} \ll R_{S}$ shows a maximum amplitude for $U_{\mathrm{G} 1}=-1.1 \mathrm{~V}$. For open circuit configuration, the measured photovoltage increases at larger negative bias voltages and achieves maximum at the threshold voltage, $U_{\text {th }}=-1.3 \mathrm{~V}$. Corresponding data will be presented and discussed below. While the non-monotonic behavior of the signal for gate voltage variation is well known for FET detectors ${ }^{1,2,42}$ the signal sign inversion upon a change of the radiation polarization, see Fig. 2(a), is generally not expected for standard Dyakonov-Shur FET detectors indicating crucial role of the lateral superlattice in the photocurrent generation. To demonstrate that the observed effect indeed stems from the lateral asymmetry of the periodic potential, we interchanged the voltages applied to the gates. Figure 2(b) shows the results obtained for zero gate voltage at the first gate and $U_{\mathrm{G} 2}=-0.9 \mathrm{~V}$ at the second one. The figure reveals that changing the sign of the lateral potential asymmetry, see insets of Figs. 2(a) and 2(b), results in the sign inversion of all contributions besides the polarization independent offset. The situation holds for almost all values of $U_{\mathrm{G} 2}$, see the insets in Figs. 2(a) and 2(b). Significantly, the proper choice of the relation between amplitudes of the individual gate potentials allows one to suppress completely one or the other photocurrent contribution. Figure 3(a) demonstrates that for close values of gate voltages the circular photocurrent vanishes (corresponding potential profile for $U_{\mathrm{G} 1}=-1.1 \mathrm{~V}$ and $U_{\mathrm{G} 2}=-0.9 \mathrm{~V}$ is shown in the inset in Fig. 3). The interplay of the contributions upon variation of $U_{\mathrm{G} 1}$ and for fixed $U_{\mathrm{G} 2}=-1.1 \mathrm{~V}$ is shown in the inset in Fig. 3(a). It is seen that for nonzero second gate voltage the circular, $j_{c}$, and linear, $j_{2}$, photocurrent contributions change their direction with increasing $U_{\mathrm{G} 1}$. Moreover, the inversions take place at different $U_{\mathrm{G} 1}$ voltages. This fact can be used to switch on and off the circular photocurrent $j_{C} \propto P_{\text {circ }}$ contribution.

To support the conclusion that $j_{1}$ and $j_{2}$ photocurrent contributions are caused by the linearly polarized light component, we carried out additional measurements applying linearly polarized light. The gate dependence of the normalized photocurrent $j_{y} / I$ measured for samples \#A and \#B for several azimuth angles $\alpha$ are shown in Fig. 4(a). The inset in this figure presents the dependence of $j_{y} / I$ on the electric field orientation. The polarization dependence is well described by Eq. (1) taking into account that for linearly polarized light the last term vanishes and the Stokes parameters are given by

$$
s_{1}(\alpha)=\cos 2 \alpha, \quad s_{2}(\alpha)=\sin 2 \alpha .
$$

Here, $\alpha=2 \beta$ defines the orientation of the polarization plane and $\beta$ is the angle between the initial polarization plane and the optical axis of the half-wave plate. The magnitudes and signs of the coefficients $j_{0}, j_{1}$, and $j_{2}$ used for the fit coincide 

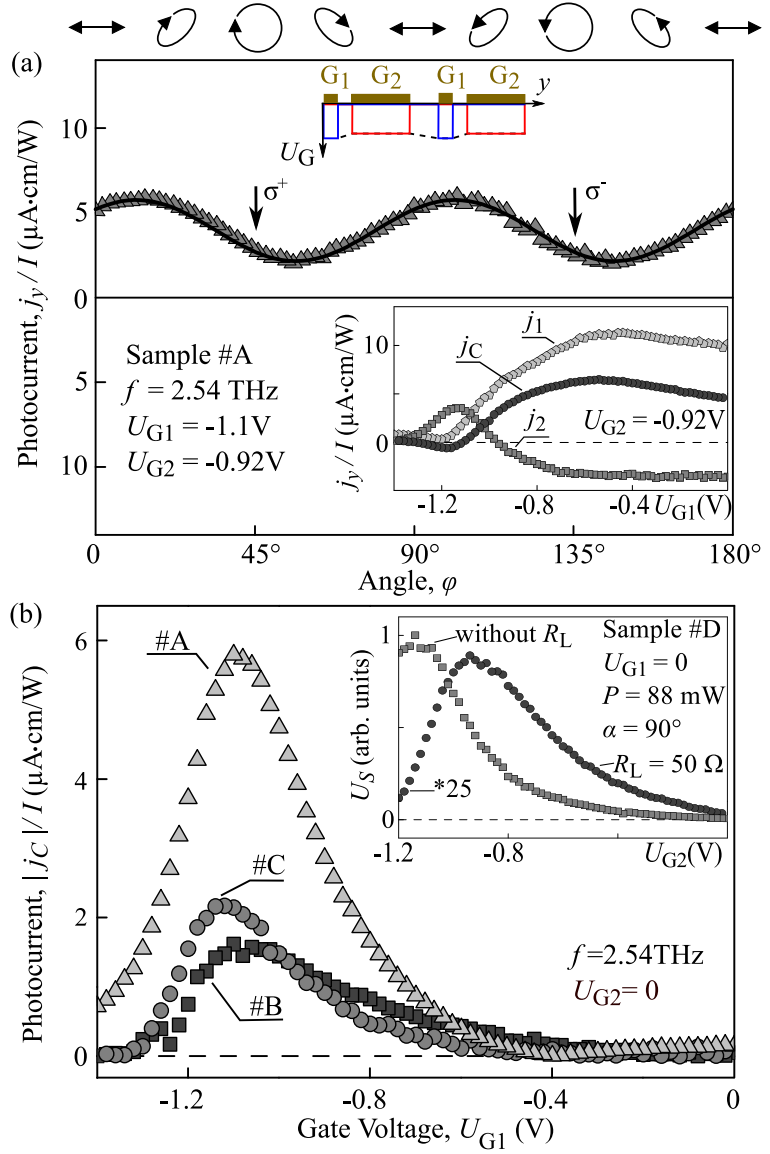

FIG. 3. (a) THz radiation induced normalized photocurrent $j_{y} / I$ as a function of the angle $\varphi$ defining the radiation helicity. The current is measured for comparable voltages applied to the first $\left(U_{\mathrm{G} 2}=-1.1 \mathrm{~V}\right)$ and the second $\left(U_{\mathrm{G} 2}=-0.92 \mathrm{~V}\right)$ gates. Full line shows fit to the total current calculated after Eq. (1). The ellipses on top illustrate the polarization states for various $\varphi$. Right inset shows amplitudes of photocurrent contributions $j_{\mathrm{C}} / I$, driven by the light helicity, and $j_{1} / I\left(j_{2} / I\right)$, induced by linear polarization, as a function of the gate voltage $U_{\mathrm{G} 1}$ or $U_{\mathrm{G} 2}$. Upper inset schematically shows corresponding gate potentials. Dashed lines are guide for the eye, indicating the potential asymmetry in $y$-direction. (b) Amplitudes of the photocurrent contributions $j_{\mathrm{C}} / I$, driven by the light helicity, as a function of the gate voltage $U_{\mathrm{G} 1}\left(U_{\mathrm{G} 2}=0\right)$ measured for three different structures \#A, \#B, and \#C. The inset shows photovoltage measured in sample \#D across $50 \Omega$ load resistance $\left(R_{L} \ll R_{S}\right)$ and directly from the sample over the lock-in amplifiers input resistance being much larger than the sample resistance $R_{s}$. Note that the former signal is multiplied by factor 25 .

with that applied for fitting of $\varphi$-dependencies obtained at the same gate voltages. These results demonstrate that photocurrents $j_{1}$ and $j_{2}$ measured in set-up applying quarter-wave plate are indeed controlled by the degree of linear polarization of elliptically polarized radiation.

The polarization sensitive photocurrent has been observed in all studied devices of similar design and arbitrary relation between second and first gate potentials. The photocurrent can always be well described by Eq. (1). Figure 3(b) summarizes the data on the helicity driven photocurrent $j_{\mathrm{C}} / I$ detected in three HEMT structures upon change of $U_{\mathrm{G} 1}$ and for $U_{\mathrm{G} 2}=0$. In all samples, we detected similar dependencies of the photocurrent characterized by close maximum positions but different signal magnitudes. The data of Fig. 3(b) as well as circles in its inset are obtained in the close circuit configuration applying $50 \Omega$ load resistance.

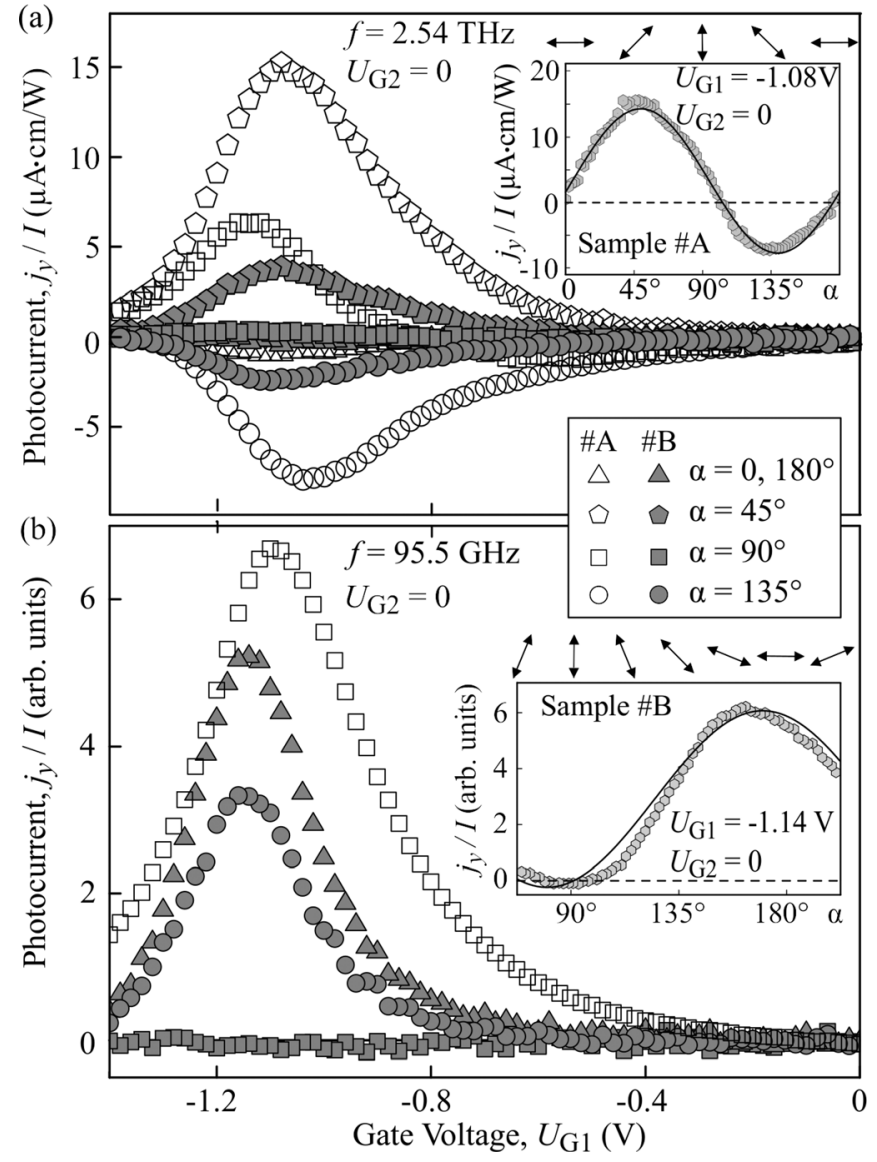

FIG. 4. (a) THz radiation induced normalized photocurrent $j_{y} / I$ excited by linearly polarized $\mathrm{THz}$ radiation in samples \#A and \#B as a function of the gate voltage $U_{\mathrm{G} 1}$. The current is shown for $U_{\mathrm{G} 2}=0$ and several in-plane orientations of the radiation electric field in respect to source-drain line defined by azimuth angles $\alpha$. Inset shows dependence of $j_{y}$ on the angle $\alpha$ obtained for $U_{\mathrm{G} 1}=-1.08 \mathrm{~V}$ and $U_{\mathrm{G} 2}=0$. Full line shows fit to the total current calculated after Eq. (6). Arrows indicate electric field orientation for several angles $\alpha$. (b) Photocurrent $j_{y} / I$ excited by linearly polarized microwave radiation $(f=95.5 \mathrm{GHz})$ in samples \#A and \#B as a function of the gate voltage $U_{\mathrm{G} 1}\left(U_{\mathrm{G} 2}=0\right)$. Inset shows dependence of $j_{y} / I$ on the azimuth angle $\alpha$ obtained in sample \#B for $U_{\mathrm{G} 1}=-1.14 \mathrm{~V}$ and $U_{\mathrm{G} 2}=0$. Full line shows fit after $j_{y} \propto \cos ^{2}(\alpha+\theta)$ with the phase angle $\theta$.

The non-monotonic behavior of the photosignal measured in this geometry is caused by the interplay of the potential asymmetry, increasing with raising second gate voltage, and raising of the sample resistance for large gate voltages. For the open circuit geometry (signal is fed to the high input impedance of lock-in amplifier), the maximum of the signal is detected for gate voltages being equal to the threshold voltage, $U_{\text {th }}$, see squares in the inset in Fig. 3(b). Following Ref. 9 , we estimate from the voltages measured in open circuit geometry the voltage responsivities for the signals corresponding to the photocurrents $j_{2}$ and $j_{C}$ as $R_{v}=U_{s} / P \times S /$ $S_{t} \approx 0.3 \mathrm{~V} / \mathrm{W}$ and $0.15 \mathrm{~V} / \mathrm{W}$, respectively. Here, $P$ the total power of the source at the detector plane, $S$ radiation beam spot area, and $S_{t}=20 \times 20 \mu \mathrm{m}^{2}$ transistor area. The voltage responsivities, being rather low as compared to that typically obtained for plasmonic FET detectors, indicate the necessity of further optimization of the structure design. Finally, we note that measurements applying microwave radiation show that for lower frequencies the polarization behavior changes 
qualitatively. Instead of the sign-alternating dependencies discussed above the signal now varies after $j_{y} \propto \cos ^{2}(\alpha+\theta)$, see inset in Fig. 4(b). This observation is in a good agreement with the Dyakonov-Shur theory ${ }^{12}$ and was reported for many conventional plasmonic FET detectors, see, e.g., Refs. 1 and 2 The gate voltage dependence of the response shown in Fig. 4 also reproduces well the results previously obtained for similar structures. ${ }^{9,43}$ Even the fact that the maximum of the signal in various structures has been obtained for different directions of the electric field vector in respect to $y$-direction (source-drain) has already been reported for these transistors and attributed to the antenna coupling of MW radiation to transistor, see Ref. 43.

\section{DISCUSSION}

The observation of the circular photocurrent and the sign-alternating linear photocurrent $j_{2}$ reveals that a microscopic process actuating these photocurrents goes beyond the plasmonic Dyakonov-Shur model typically applied to discuss operation of FET $\mathrm{THz}$ detectors. Indeed, as addressed above, the latter implies an oscillating electric field along source-drain direction ( $y$-direction) yielding sign conserving variation upon rotation of polarization plane, $j_{y} \propto \cos ^{2} \alpha .{ }^{44}$ As recently shown in Refs. 45 and 46, the Dyakonov-Shur model in fact may result in the circular photocurrent but only due to interference effects of two different channels and two interacting antennas in small size special design FETs - the model which can hardly be applied to the large DGG samples used in our experiments. At the same time, the observed polarization behavior is characteristic for the electronic ratchet effects excited in asymmetric periodic structures ${ }^{23-26}$ and linear/circular plasmonic ratchet effects. $^{22,27}$ The ratchet currents arise due to the phase shift between the periodic potential and the periodic light electric field resulting from near field diffraction in a system with broken symmetry. Microscopic theory developed in Ref. 25 shows that the helicity dependent photocurrent appears because the carriers in the laterally modulated quantum wells move in two directions and are subjected to the action of the two-component electric field. Symmetry analysis of the photocurrent shows that in our DDG structures described by $\mathrm{C}_{1}$ point group symmetry, ${ }^{47-49}$ it varies with radiation polarization after Eq. (1), being in agreement with experimental observation shown in Figs. 2-4(a). Moreover, as the ratchet photocurrents are proportional to the degree of the in-plane asymmetry, they reverse the sign upon inversion of static potential asymmetry. Exactly this behavior has been observed in experiment, see Figs. 2(a) and 2(b). The proportionality to the degree of lateral asymmetry also explains the increase of the signal with raising voltage applied to one gate at constant voltage by the other. The interplay of the degree of lateral asymmetry and periodic modulation of $\mathrm{THz}$ electric field results in the complex gate-voltage dependence, in particular, for $U_{\mathrm{G} 1} \approx U_{\mathrm{G} 2}$. As the different individual contributions to the total current effect might imply different microscopic mechanisms of the photocurrent formation, their behavior upon change of external parameters can distinct from each other. This would result in a sign-alternating gatevoltage behavior, in particular, for the range of comparable $U_{\mathrm{G} 1}$ and $U_{\mathrm{G} 2}$, like it is observed in experiment, see Fig. 2(c). While all qualitative features of the observed phenomena can be rather good described in terms of ratchet effects we would like to address another possible effect, which might trigger the helicity-driven photocurrent. It could be the differential plasmonic drag effect in the two-dimensional structure with an asymmetric double-grating gate considered in Refs. 22 and 50. As shown in Ref. 22 for a periodic AlAs/ InGaAs/InAlAs/InP structure and linearly polarized $\mathrm{THz}$ radiation, photon drag effect can be comparable in strength with the plasmonic ratchet effect at $\mathrm{THz}$ frequencies. As the circular photon drag effect has been observed in different low dimensional materials, ${ }^{36,51}$ we can expect that modification of the theory developed in Ref. 22 can also yield helicity driven plasmonic drag current compatible with the ratchet one.

Finally, we note that the ratchet effects (either electronic or plasmonic) can be greatly increased due to the resonant enhancement of the near-field in two-dimensional electron system at the plasmon resonance excitation as it was shown for the plasmonic ratchet in Refs. 20 and 27. The resonant plasmon condition $\omega \tau>1$ (see Ref. 12) can be well satisfied in our structure ( $\omega \tau=4$ at $2.54 \mathrm{THz})$. As shown in Ref. 20, the fundamental plasmon resonance is excited in a similar structure at frequency around $2 \mathrm{THz}$. Therefore, the plasmon resonance excitation can contribute to the observed ratchet effects independently of particular microscopic mechanisms of the ratchet photocurrent formation. The measurements in a broader $\mathrm{THz}$ frequency range could elucidate the role of the plasmonic resonance excitation in the ratchet photocurrent enhancement.

\section{SUMMARY}

To summarize, our measurements demonstrate that dual-grating-gate InAlAs/InGaAs/InAlAs/InP high electron mobility transistors excited by terahertz radiation can yield a helicity sensitive photocurrent response at $\mathrm{THz}$ frequencies. We show that HEMTs with asymmetric lateral superlattice of gate fingers with unequal widths and spacing can be applied for generation of a photocurrent defined by linearly and circularly radiation polarization components. Moreover, one can obtain photoresponse being proportional to one of the Stokes parameters simply by variation of voltages applied to the individual gates. The photocurrent formations can be well described in terms of ratchet effects excited by terahertz radiation. By that the lateral grating induces a periodical lateral potential acting on the $2 \mathrm{D}$ electron gas in QW. This grating also modulates the incident radiation in the near field and hence in the plane of the 2DES, resulting in circular, linear, and polarization-independent ratchet effects. While the responsivity of the polarization dependent response is lower than that reported for FET transistors, it can be substantially improved by optimization of the structure design leading the resonant enhancement of the ratchet effects the plasmon resonance excitation. 


\section{ACKNOWLEDGMENTS}

We thank V. Popov for helpful discussions. The financial support from the DFG (SFB 689) is gratefully acknowledged.

${ }^{1}$ W. Knap and M. Dyakonov, "Plasma wave THz detectors and emitters," in Handbook of Terahertz Technology, edited by D. Saeedkia (Woodhead Publishing, Waterloo, Canada, 2013), pp. 121-155.

${ }^{2}$ W. Knap, S. Rumyantsev, M. S. Pea Vitiello, D. Coquillat, S. Blin, N. Dyakonova, M. Shur, F. Teppe, A. Tredicucci, and T. Nagatsuma, Nanotechnology 24, 214002 (2013).

${ }^{3}$ S. Boppel, A. Lisauskas, A. Max, V. Krozer, and H. G. Roskos, Opt. Lett. 37, 536 (2012).

${ }^{4}$ V. M. Muravev and I. V. Kukushkin, Appl. Phys. Lett. 100, 082102 (2012).

${ }^{5}$ M. Tonouchi, Nat. Photonics 1, 97 (2007).

${ }^{6}$ F. Schuster, D. Coquillat, H. Videlier, M. Sakowicz, F. Teppe, L. Dussopt, B. Giffard, T. Skotnicki, and W. Knap, Opt. Express 19, 7827 (2011).

${ }^{7}$ G. C. Dyer, S. Preu, G. R. Aizin, J. Mikalopas, A. D. Grine, J. L. Reno, J. M. Hensley, N. Q. Vinh, A. C. Gossard, M. S. Sherwin, S. J. Allen, and E. A. Shaner, Appl. Phys. Lett. 100, 083506 (2012).

${ }^{8}$ S. Preu, M. Mittendorff, S. Winnerl, H. Lu, A. C. Gossard, and H. B. Weber, Opt. Express 21, 17941 (2013).

${ }^{9}$ T. Watanabe, S. A. Boubanga-Tombet, Y. Tanimoto, D. Fateev, V. Popov, D. Coquillat, W. Knap, Y. M. Meziani, Y. Wang, H. Minamide, H. Ito, and T. Otsuji, IEEE Sens. J. 13, 89 (2013).

${ }^{10}$ D. B. But, C. Drexler, M. V. Sakhno, N. Dyakonova, O. Drachenko, F. F. Sizov, A. Gutin, S. D. Ganichev, and W. Knap, J. Appl. Phys. 115, 164514 (2014).

${ }^{11}$ W. Knap, D. B. But, N. Dyakonova, D. Coquillat, A. Gutin, O. Klimenko, S. Blin, F. Teppe, M. S. Shur, T. Nagatsuma, S. D. Ganichev, and T. Otsuji, Recent Results on Broadband Nanotransistor Based $\mathrm{THz}$ Detectors, NATO Science for Peace and Security Series B, Physics and Biophysics: THz and Security Applications, edited by C. Corsi and F. Sizov (Springer, Dordrecht, Netherlands, 2014), pp.189-210.

${ }^{12}$ M. Dyakonov and M. S. Shur, IEEE Trans. Electron. Dev. 43(3), 380 (1996).

${ }^{13}$ W. Knap, Y. Deng, S. Rumyantsev, J.-Q. Lu, M. S. Shur, C. A. Saylor, and L. C. Brunel, Appl. Phys. Lett. 80, 3433 (2002).

${ }^{14}$ W. Knap, V. Kachorovskii, Y. Deng, S. Rumyantsev, J.-Q. Lu, R. Gaska, M. S. Shur, G. Simin, X. Hu, M. Asif Khan, C. A. Saylor, and L. C. Brunel, J. Appl. Phys. 91, 9346 (2002).

${ }^{15}$ T. Otsuji, M. Hanabe, T. Nishimura, and E. Sano, Opt. Express 14, 4815 (2006).

${ }^{16}$ S. Sassine, Yu. Krupko, J.-C. Portal, Z. D. Kvon, R. Murali, K. P. Martin, G. Hill, and A. D. Wieck, Phys. Rev. B 78, 045431 (2008).

${ }^{17}$ D. Coquillat, S. Nadar, F. Teppe, N. Dyakonova, S. Boubanga-Tombet, W. Knap, T. Nishimura, T. Otsuji, Y. M. Meziani, G. M. Tsymbalov, and V. V. Popov, Opt. Express 18, 6024 (2010).

${ }^{18}$ V. V. Popov, J. Infrared Millimeter THz Waves 32, 1178 (2011).

${ }^{19}$ G. C. Dyer, G. R. Aizin, J. L. Reno, E. A. Shaner, and S. J. Allen, IEEE J. Sel. Top. Quantum Electron. 17, 85 (2011).

${ }^{20}$ V. V. Popov, D. V. Fateev, T. Otsuji, Y. M. Meziani, D. Coquillat, and W. Knap, Appl. Phys. Lett. 99, 243504 (2011).

${ }^{21}$ E. S. Kannan, I. Bisotto, J.-C. Portal, T. J. Beck, and L. Jalabert, Appl. Phys. Lett. 101, 143504 (2012).

${ }^{22}$ V. V. Popov, Appl. Phys. Lett. 102, 253504 (2013).

${ }^{23}$ P. Olbrich, E. L. Ivchenko, T. Feil, R. Ravash, S. D. Danilov, J. Allerdings, D. Weiss, and S. D. Ganichev, Phys. Rev. Lett. 103, 090603 (2009).

${ }^{24}$ E. L. Ivchenko and S. D. Ganichev, JETP Lett. 93, 673 (2011).

${ }^{25}$ P. Olbrich, J. Karch, E. L. Ivchenko, J. Kamann, B. Maerz, M. Fehrenbacher, D. Weiss, and S. D. Ganichev, Phys. Rev. B 83, 165320 (2011).

${ }^{26}$ A. V. Nalitov, L. E. Golub, and E. L. Ivchenko, Phys. Rev. B 86, 115301 (2012).

${ }^{27}$ I. V. Rozhansky, V. Yu. Kachorovskii, and M. S. Shur, Phys. Rev. Lett. 114, 246601 (2015)

${ }^{28}$ B. E. A. Saleh and M. C. Teich, Fundamentals of Photonics (John Wiley \& Sons, New York, 2003).

${ }^{29}$ S. D. Ganichev, E. L. Ivchenko, and W. Prettl, Physica E 14, 166 (2002).
${ }^{30}$ S. N. Danilov, B. Wittmann, P. Olbrich, W. Eder, W. Prettl, L. E. Golub, E. V. Beregulin, Z. D. Kvon, N. N. Mikhailov, S. A. Dvoretsky, V. A. Shalygin, N. Q. Vinh, A. F. G. van der Meer, B. Murdin, and S. D. Ganichev, J. Appl. Phys. 105, 013106 (2009).

${ }^{31}$ S. D. Ganichev, J. Kiermaier, W. Weber, S. N. Danilov, D. Schuh, Ch. Gerl, W. Wegscheider, D. Bougeard, G. Abstreiter, and W. Prettl, Appl. Phys. Lett. 91, 091101 (2007).

${ }^{32}$ S. D. Ganichev, W. Weber, J. Kiermaier, S. N. Danilov, D. Schuh, W. Wegscheider, Ch. Gerl, D. Bougeard, G. Abstreiter, and W. Prettl, J. Appl. Phys. 103, 114504 (2008).

${ }^{33}$ D. Nguyen, K. Hogan, A. Blew, and M. Cordes, J. Cryst. Growth 272, 59 (2004).

${ }^{34}$ T. Enoki, H. Yokoyama, Y. Umeda, and T. Otsuji, Jpn. J. Appl. Phys., Part 1 37, 1359 (1998).

${ }^{35}$ S. D. Ganichev, S. A. Tarasenko, V. V. Bel'kov, P. Olbrich, W. Eder, D. R. Yakovlev, V. Kolkovsky, W. Zaleszczyk, G. Karczewski, T. Wojtowicz, and D. Weiss, Phys. Rev. Lett. 102, 156602 (2009).

${ }^{36}$ J. Karch, P. Olbrich, M. Schmalzbauer, C. Zoth, C. Brinsteiner, M. Fehrenbacher, U. Wurstbauer, M. M. Glazov, S. A. Tarasenko, E. L. Ivchenko, D. Weiss, J. Eroms, R. Yakimova, S. Lara-Avila, S. Kubatkin, and S. D. Ganichev, Phys. Rev. Lett. 105, 227402 (2010).

${ }^{37}$ S. D. Ganichev, Physica B 273-274, 737-742 (1999).

${ }^{38}$ J. Karch, C. Drexler, P. Olbrich, M. Fehrenbacher, M. Hirmer, M. M. Glazov, S. A. Tarasenko, E. L. Ivchenko, B. Birkner, J. Eroms, D. Weiss, R. Yakimova, S. Lara-Avila, S. Kubatkin, M. Ostler, T. Seyller, and S. D. Ganichev, Phys. Rev. Lett. 107, 276601 (2011).

${ }^{39}$ M. M. Glazov and S. D. Ganichev, Phys. Rep. 535, 101 (2014).

${ }^{40}$ S. D. Ganichev and W. Prettl, Intense Terahertz Excitation of Semiconductors (Oxford University Press, Oxford, 2006).

${ }^{41}$ While being detected in all reported measurements, a polarization independent offset given by the coefficient $j_{0}$ will not be discussed in detail. Instead, hereafter we focus on helicity sensitive photocurrent, $j_{\mathrm{C}}$, and currents driven by linearly polarized light, $j_{1}$ and $j_{2}$.

${ }^{42}$ M. Sakowicz, M. B. Lifshits, O. A. Klimenko, F. Schuster, D. Coquillat, F. Teppe, and W. Knap, J. Appl. Phys. 110, 054512 (2011).

${ }^{43}$ D. Coquillat, V. Nodjiadjim, A. Konczykowska, M. Riet, N. Dyakonova, C. Consejo, F. Teppe, J. Godin, and W. Knap, in Digest of International Conference on Infrared, Millimeter, and Terahertz Waves, Tucson, USA, 2014.

${ }^{44}$ Note that signal variation with polarization is, apart the offset, identical with that of $s_{1}$, therefore this polarization dependence can also be used to describe the $j_{1}$-related photocurrent behavior.

${ }^{45}$ C. Drexler, N. Dyakonova, P. Olbrich, J. Karch, M. Schafberger, K. Karpierz, Yu. Mityagin, M. B. Lifshits, F. Teppe, O. Klimenko, Y. M. Meziani, W. Knap, and S. D. Ganichev, J. Appl. Phys. 111, 124504 (2012).

${ }^{46}$ K. S. Romanov and M. I. Dyakonov, Appl. Phys. Lett. 102, 153502 (2013).

${ }^{47}$ All previous works aimed to the radiation induced ratchet effects discuss the case of unconnected parallel metal stripes: a system belonging to Cs point group symmetry consisting of the identity element and the reflection in the plane perpendicular to the stripes. ${ }^{22-27}$ For this symmetry, circular photocurrent $j_{C}$ and the photocurrent $j_{2}$ can be generated along stripes only whereas polarization independent offset $j_{0}$ and photocurrent $j_{1}$ are allowed in the perpendicular to that direction (source-drain). Design of our DDG structures with interconnected metal stripes in each of gates excludes reflection plane reducing the point group symmetry to $C_{1}$. As a result, the symmetry does not imply any restrictions and the photocurrent includes all four individual contributions $\left(j_{0}, j_{1}, j_{2}\right.$ and $\left.j_{C}\right)$ which are allowed in any inplain direction. More details on the symmetry analysis of photocurrents in quantum wells of $\mathrm{C}_{1}$ symmetry can be found in Refs. 48 and 49.

${ }^{48}$ B. Wittmann, S. N. Danilov, V. V. Bel'kov, S. A. Tarasenko, E. G. Novik, H. Buhmann, C. Brüne, L. W. Molenkamp, E. L. Ivchenko, Z. D. Kvon, N. N. Mikhailov, S. A. Dvoretsky, N. Q. Vinh, A. F. G. van der Meer, B. Murdin, and S. D. Ganichev, Semicond. Sci. Technol. 25, 095005 (2010).

${ }^{49}$ V. V. Bel'kov and S. D. Ganichev, Semicond. Sci. Technol. 23, 114003 (2008).

${ }^{50}$ V. V. Popov, D. V. Fateev, E. L. Ivchenko, and S. D. Ganichev, Phys. Rev. B 91, 235436 (2015).

${ }^{51}$ V. A. Shalygin, H. Diehl, Ch. Hoffmann, S. N. Danilov, T. Herrle, S. A. Tarasenko, D. Schuh, Ch. Gerl, W. Wegscheider, W. Prettl, and S. D. Ganichev, JETP Lett. 84, 570 (2007). 